\title{
Postpolio syndrome: a 5 year follow-up
}

\author{
Johan Kvalvik Stanghelle and Lillian V Festvåg \\ Sunnaas Rehabilitation Hospital, 1450 Nesoddtangen, Norway
}

\begin{abstract}
The purpose of this investigation was to study subjective symptoms, medical and social situation, pulmonary function and physical work capacity during a period of $3-5$ years in patients with the postpolio syndrome. We evaluated 68 patients consecutively admitted to our hospital because of postpolio syndrome, and re-evaluated 63 of the same patients $3-5$ years later, 43 women and 20 men with mean age $55 \pm 10$ (1 SD) years at the second evaluation. The patients answered a questionnaire about their subjective symptoms and medical and social situation, and underwent spirometry as well as symptom-limited exercise stress testing. Most patients experienced increasing symptoms and physical disability related to their polio, while the majority reported that their mental health were unchanged or improved. The lung function was in average moderately reduced of restrictive type, and only minor changes were found during the 3-5 years. A pronounced reduction in peak oxygen uptake was seen at the first evaluation, especially in women. At the second examination, peak oxygen uptake was further decreased, especially in men, more than predicted from increasing age. The patients increased their body mass index significantly during the same period. These results indicate that subjective symptoms and physical disability related to polio increased with increasing age in these patients with the post-polio syndrome, and cardiorespiratory deconditioning and weight gain also became increasing problems in most patients. However, the mental status of the patients remained stable or improved, possibly due to our comprehensive re-rehabilitation and educational programme.
\end{abstract}

Keywords: poliomyelitis; postpolio syndrome; rehabilitation, lung function; exercise testing

\section{Introduction}

In a previous study ${ }^{1}$ we presented a study of pulmonary function and symptom-limited exercise stress testing in 68 consecutive patients with the postpolio syndrome admitted to our rehabilitation hospital, concluding that cardiorespiratory deconditioning was considerable in most of these subjects. In the present investigation we performed identical lung function and exercise stress tests in the same subjects 3-5 years later, in order to study whether their pulmonary function and physical work capacity had deteriorated during this period, eventually more than predicted with increasing age in healthy persons. ${ }^{2}$

These postpolio patients had undergone a comprehensive rehabilitation programme with interdisciplinary evaluation, education and adaptations at our hospital. ${ }^{3,4}$ We therefore also wanted to study whether they, in spite of this intervention, had further changes in subjective symptoms, medical and social situation.

Correspondence: JK Stanghelle

\section{Material}

Sixty-eight patients were included, consecutively admitted to Sunnaas Rehabilitation Hospital over a period of 3 years with the presumptive postpolio syndrome according to criteria described by Halstead et al with (a) a prior episode of paralytic polio; (b) a period of neurological recovery followed by an extended interval of functional stability (at least 20 years); (c) the gradual or abrupt onset of new neurogenic (non-disuse) weakness in previously affected and/or unaffected muscles, accompanied or not by other new health problems such as excessive fatigue, muscle pain, joint pain, decreased endurance, decreased function, and atrophy, and (d) exclusion of medical, orthopedic, and neurological conditions that might cause the new health problems. ${ }^{5,6}$ Sixty-three of these patients were re-evaluated $3-5$ years (in average 4 years) later; 43 women and 20 men with mean age $55 \pm 10$ (1 SD) years at the second evaluation. Characteristics of the subjects are presented in Table 1. Of the five patients not participating in the followup study one had died, one was admitted to a nursing home, three declined to enter the study, and one patient was excluded due to additional cognitive problems. 
Postpolio syndrome at $\mathbf{5}$ years

JK Stanghelle and LV Festvåg
Table 1 Characteristics for 62 patients with the postpolio syndrome participating in the study. Values are given in mean \pm 1 SD

\begin{tabular}{lcc}
\hline & Examination 1 & Examination 2 \\
\hline Age (years) & $51 \pm 10$ & $55 \pm 10$ \\
Height $(\mathrm{cm})$ & $165 \pm 10$ & $164 \pm 11$ \\
Weight $(\mathrm{kg})$ & $65 \pm 15$ & $69 \pm 15^{*}$ \\
BMI (weight $/$ height $^{2}$ ) & $24 \pm 5$ & $26 \pm 5^{*}$ \\
\hline
\end{tabular}

$\mathrm{BMI}=$ body mass index $=$ body weight $/$ height $^{2}$ (reference values $19-25)$. *significant changes between examination 1 and $2, P<0.05$

\section{Methods}

After obtaining informed consent, all patients answered a questionnaire about their subjective symptoms and medical and social situation, especially regarding changes during the period between the first and the second examination. The questionnaire was supplemented by an interview by a medical doctor and a physiotherapist.

The patients thereafter performed lung function and exercise stress testing, identical procedures with those of the first examination. ${ }^{1}$ Spirometry was performed by dry spirometry (Vitalograph Compact, Vitalograph Ltd, Birmingham, England). The measured forced vital capacity (FVC), forced expiratory volume for one second (FEV1), and maximal voluntary ventilation (MVV) were compared with predicted values for sex, height and age in healthy persons. ${ }^{7}$

Exercise testing was performed either with arm $(n=24)$ or leg $(n=38)$ bicycle ergometer (Jaeger, Würzburg, Germany). The protocols for the individual subjects were supposed to be identical with their exercise test $3-5$ years earlier. ${ }^{1}$ The subjects who performed arm ergometry pedalled at intervals of $3 \mathrm{~min}$ with a 2 min pause between the intervals. Most of these subjects started with a workload of $10 \mathrm{~W}$, with increases of $20 \mathrm{~W}$ for every interval. Subjects who performed leg ergometry usually started with a workload of $25 \mathrm{~W}$, which increased by 25 or $50 \mathrm{~W}$ every interval of $3 \mathrm{~min}$, without pauses, until exhaustion. The subjects rated their subjective perceived exertion at the end of the exercise test according to the Borg scale. ${ }^{8}$ Oxygen uptake $(\dot{V} \mathrm{O} 2)$, ventilation $(\dot{V})$ and respiratory quotient $(\mathrm{R})$ were measured every $30 \mathrm{~s}$ during exercise (Jaeger EOS Spring Exercise System, Würzburg, Germany). Peak $\dot{V} \mathrm{O} 2$ was labelled maximal $\dot{V} \mathrm{O} 2$ $(\max \dot{V} \mathrm{O} 2)$, even if some subjects did not reach the criteria for max $\dot{V} \mathrm{O} 2 .^{2}$ The capillary concentration of lactate was measured (Model 23L Lactate Analyser, Yellow Springs Instrument, USA) 3 min after the end of the exercise.

Max $\dot{V} \mathrm{O} 2$ was compared with predicted average values for age and sex in healthy persons. ${ }^{2}$ Predicted max $\dot{V} \mathrm{O} 2$ with arm ergometry was presumed to be $70 \%$ of predicted max $\dot{V} \mathrm{O} 2$ with leg ergometry. ${ }^{2}$

Heart rate (HR) was followed by continuous electrocardiogram recording (Cardiac Monitor 573,
Kone, USA). Registered maximal HR (max HR) was compared with predicted maximal HR (pred max HR) for age, using the formula: 220 beats/min minus the subject's age $=$ pred $\max \mathrm{HR}^{2}$

\section{Statistics}

The results are given in mean \pm 1 SD and range. Comparisons between groups were obtained using Student's $t$-test, and Fischers's exact test for proportions between groups. The criterion for statistical significance was $P<0.05$.

\section{Results}

Pulmonary function values at the first and the second examination are shown in Table 2, indicating that the subjects had on average a slightly reduced lung function of restrictive type, with only minor changes between the two tests. However, the number of patients with FEV1 below 1,5 1 increased from six to ten, but none of them had FEV1 below $30 \%$ of predicted values.

Table 3 shows the cardiopulmonary values at peak exercise in subjects who exercised with arm and leg ergometry, during the first and second test, respectively, Max $\dot{V} \mathrm{O} 2$ was on average severely reduced at test 1, especially in women, and the values $3-5$ years later were significantly lower, also when given in percentage of predicted values, indicating that the

Table 2 Lung function values for 62 patients with the postpolio syndrome, during two tests with $3-5$ years interval. (Test 1 and Test 2, respectively). Values are given in mean $\pm 1 \mathrm{SD}$

\begin{tabular}{lll}
\hline & Test 1 & Test 2 \\
\hline FVC (\% pred) & $93 \pm 24$ & $92 \pm 23$ \\
FEV1 (\% pred) & $84 \pm 22$ & $84 \pm 23$ \\
FEV1/FVC (\% pred) & $74 \pm 7$ & $74 \pm 9$ \\
MVV (\% pred) & $86 \pm 25$ & $90 \pm 25$ \\
\hline
\end{tabular}

*significant changes between examination 1 and $2, P<0.05$

Table 3 Values at maximal exercise, during two tests with $3-5$ years interval (Test 1 and Test 2, respectively). Values are given in mean $\pm 1 \mathrm{SD}$

\begin{tabular}{|c|c|c|}
\hline & Test 1 & Test 2 \\
\hline Max load (watt) & $84 \pm 49$ & $82 \pm 48$ \\
\hline Peak V்O2 (1/min) & $1.3 \pm 0.6$ & $1.2 \pm 0.5^{*}$ \\
\hline Peak vंO2 $(\mathrm{ml} / \mathrm{kg} / \mathrm{min})$ & $20.1 \pm 7.5$ & $17.6 \pm 6.0^{* *}$ \\
\hline Peak $\dot{\mathrm{VO}} 2$ (\% pred) & $64 \pm 20$ & $57 \pm 15^{* *}$ \\
\hline Max HR & $158 \pm 20$ & $152 \pm 20^{*}$ \\
\hline Max V/MVV & $58 \pm 18$ & $52 \pm 14$ \\
\hline $\operatorname{Max} \mathrm{R}$ & $1.15 \pm 0.13$ & $1.21 \pm 0.19$ \\
\hline Max lactate & $5.9 \pm 2.0$ & $5.9 \pm 2.4$ \\
\hline Max RPE (Borg scale) & $18 \pm 2$ & $18 \pm 2$ \\
\hline
\end{tabular}

*significant changes between examination 1 and $2, P<0.05$. **significant changes between examination 1 and $2, P<0.005$ 
deterioration was more pronounced than expected with increasing age. Max $\dot{V O} 2$ decreased more pronounced in men that in women during the follow up period.

At test 2, we found that 23 patients had $\max \dot{V} \mathrm{O} 2$ below $50 \%, 22$ patients were between 50 and $70 \%$, and 15 patients above $70 \%$ of predicted values. In two patients the results were useless due to technical reasons. None of the subjects had max $\dot{V} \mathrm{O} 2$ above $100 \%$ of predicted values at the second evaluation.

The maximal values of $\mathrm{HR}, \dot{V} / \mathrm{MVV}, \mathrm{R}$, lactate concentration and subjective rating according to Borgs scale indiated that the results at test 1 and 2 were comparable, and also that the patients in average had exerted themselves close to their maximum, above the anaerobic threshold ${ }^{2}$ (Table 3).

Figure 1 shows how the patients themselves reported eventual changes in their physical and mental health during the follow-up period. Totally 38 patients $(60 \%), 24$ women and 14 men (NS), reported a worsening in their physical health. More women than men, 13 versus one $(P<0.02)$, reported improvement in physical health. Fifteen patients $(24 \%)$ reported a worse mental health at the second evaluation, while 20 patients $(32 \%), 19$ women versus one man $(P<0.002)$, stated that their mental health was improved during the follow-up period (Figure 1).

A total of 16 patients $(26 \%)$ reported that they had experienced an intercurrent or complicating disease besides their polio during the same period.

Figure 2 shows the percentage of patients who were claiming on increased health problems related to their polio during the last $3-5$ years. Between 60 and $77 \%$

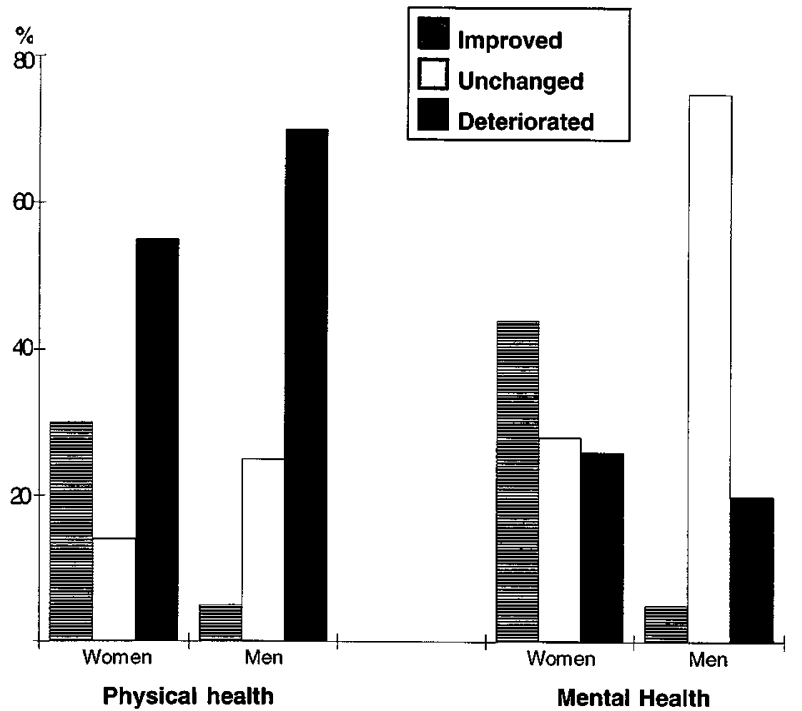

Figure 1 Subjective changes in physical and mental health during 3-5 years in 43 women and 20 men with the postpolio syndrome. The values for women and men, respectively, are given in percentage of patients who participated in the study had experienced increased symptoms, such as muscle weakness, general fatigue, cold intolerance, pain in muscles/joints and reduced mobility (Figure 2). In addition, less common symptoms were also reported to be increased, such as breathing problems (33\%), muscle atrophy $(33 \%)$ and swallowing disorders $(11 \%)$

Figure 3 shows the patients' subjective rating of disability; in the acute phase of poliomyelitis, in the stable phase, and at examination 1 and 2, respectively. The results show an increased proportion of persons who reports severe and moderate disability, and a decreased proportion of persons with mild disability; during the period from the stable phase to examination 1, and a further worsening during the period between examination 1 and 2 (Figure 3).

Figure 4 illustrates the increased use of technical aids and assistive devices in the follow-up period, showing that a considerable number of the patients had taken into use electrical or manual wheelchairs,

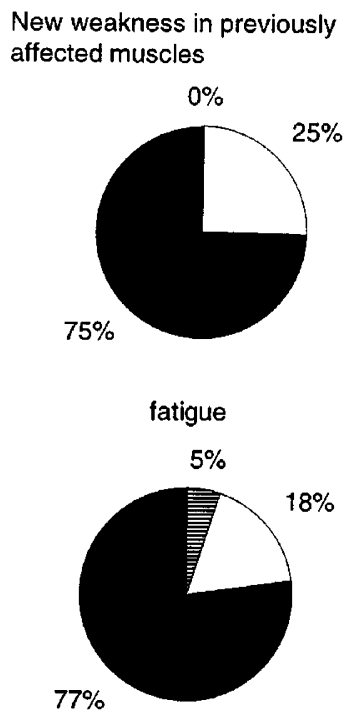

New weakness in previously "not affected muscles"
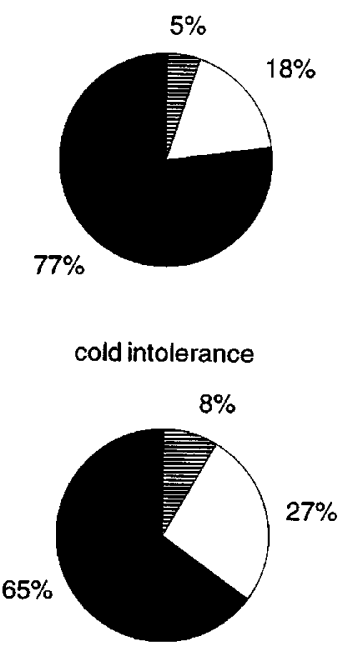

muscle- and joint pain

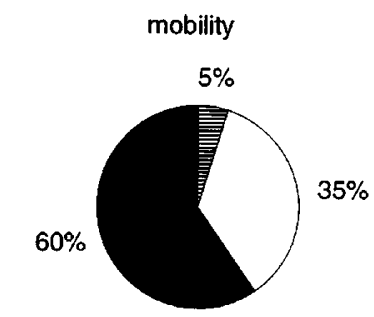

unaffected

unchanged

deteriorated

Figure 2 Subjective changes, according to the patients' own statements, in physical symptoms during 3-5 years in 63 patients with the postpolio syndrome 


$\square_{\text {Mild }} \square_{\text {Moderate }} \square_{\text {Severe }}$

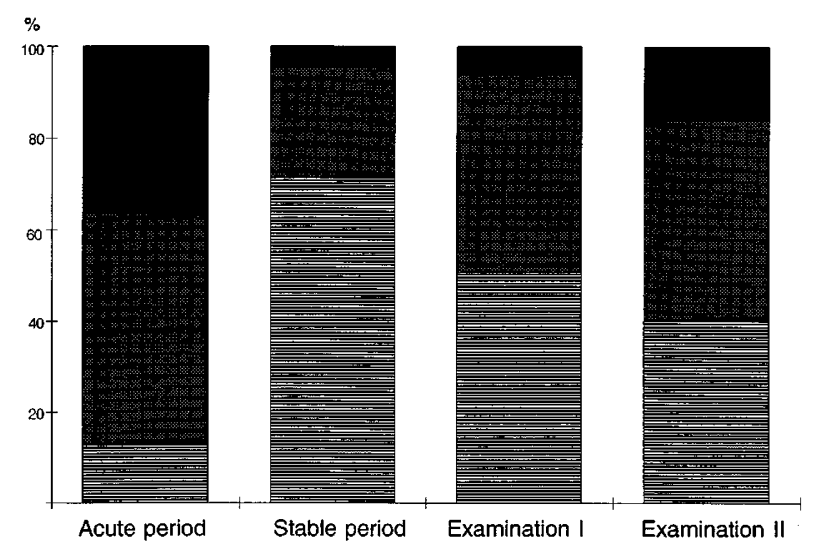

Figure 3 Subjective rating of general physical impairment, according to the patients' own statements about their general physical condition, categorized into mild, moderate or severe physical impairment; in acute and stable periods, and at examination 1 and 2, respectively. The ratings of impairment during the different phases were made retrospectively at examination 2

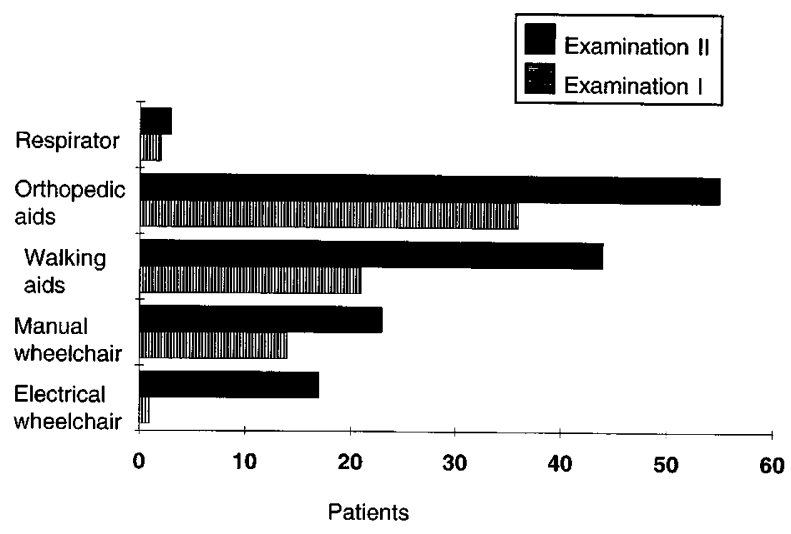

Figure 4 Number of patients with the postpolio syndrome using respirator, orthopedic aids, walking aids, manual wheelchair and electrical wheelchair examination 1 and 2 , respectively

walking and orthopaedic aids. Patients using respirator increased from three to four during the last 3-5 years (Figure 4).

Figure 5 shows the patients' need of personal assistance. Dependence in personal activities of daily living increased from two to six patients, ie $10 \%$ of this material at examination 2 , while a majority of the postpolio patients was in need of personal assistance for instrumental activities of daily living, such as housework and carrying things at examination 2 (Figure 5).

Figure 6 illustrates the situation of employment for the postpolio patients at examination 1 and 2, showing

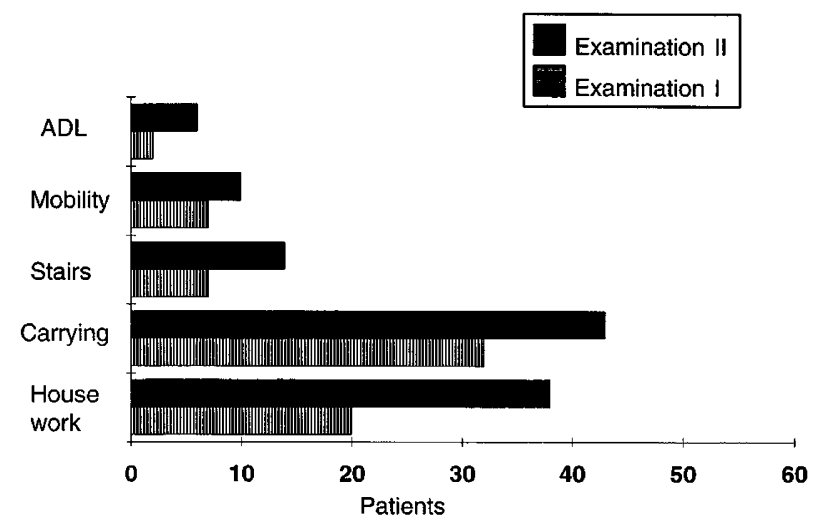

Figure 5 Number of patients with the postpolio syndrome in need of personal assistance at examination 1 and 2, respectively. The numbers indicate patients with total inability to perform the given activities without personal assistance

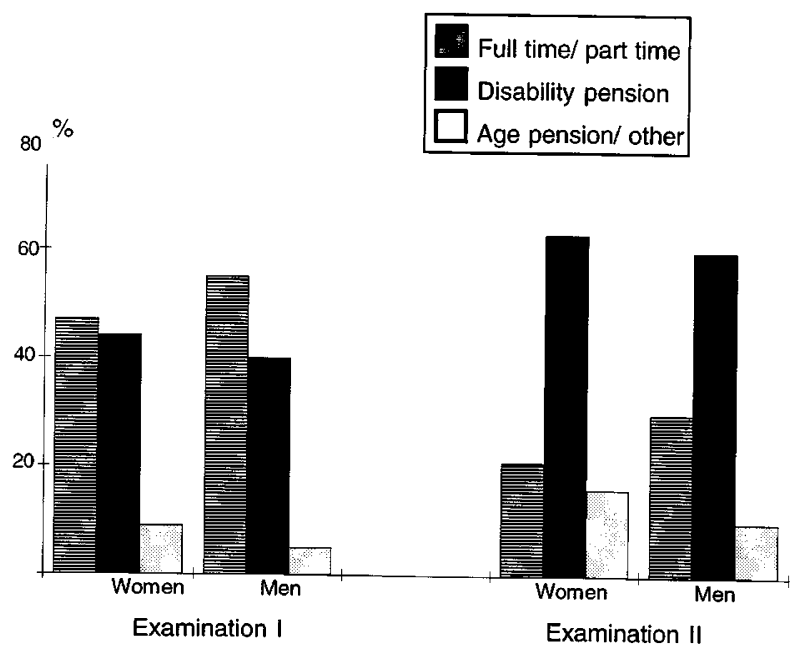

Figure 6 Employment situation among 43 women and 20 men with the postpolio syndrome at examination 1 and 2, respectively

that the number of subjects still in job, full or part time, had decreased from $31(50 \%)$ to $17(27 \%)$ during $3-5$ years, with a corresponding increase in persons who received disability pension. More women than men $(23$ versus $7, P<0.05)$ were on disability pension at the second evaluation (Figure 6).

A majority of these patients $(74 \%)$ stated that they had changed their routines at home due to their deterioration during the last $3-5$ years, $85 \%$ reported that they rested more/more often, $45 \%$ said that they received more help at home, and $45 \%$ of those in job stated that they had changed their working tasks due to their polio problems. Even if $45 \%$ reported that they had reduced their social activity, only 9\% (five subjects) had experienced a change in their marital status during the follow-up period. 
Even if the level of physical activity was reduced in most patients, totally $68 \%$ still reported to do some kind of exercise by their own or by help of a physiotherapist at least once a week, and $40 \%$ exercised several times weekly. However, the exact amount of exercise duration, intensity and frequency are lacking.

\section{Discussion}

The principle findings of this investigation was a further deterioration of cardiorespiratory fitness combined with a further increase of subjective health problems and disability in patients with the postpolio syndrome during a period of 3-5 years. In spite of a comprehensive, interdisciplinary evaluation and intervention programme due to their new problems, there was a surprisingly high percentage of the patients who still experienced increased symptoms and disability, with more need for technical aids, assistive devices and personal assistance for activites of daily living (Figures 1-5). It must be emphasized, however, that this material was selected to involve patients who already fulfilled the criteria for postpolio syndrome when they were referred to the national rehabilitation hospital after a stable phase of $36 \pm 8$ years and with $6 \pm 4$ years since the new symptoms appeared. ${ }^{1}$ Thus, these patients had possibly extensive problems compared with the polio population in general.

One of the main purposes of this study was to measure the cardiorespiratory fitness $3-5$ years later in patients with the postpolio syndrome. Our findings show that the working capacity deteriorated more than expected in healthy persons with increasing age, partly related to increased body weight (Tables 1 and 3 ). Thus, the lower aerobic capacity combined with higher body weight might contribute to the increasing poliorelated symptoms and disability. According to normal physiological principles, the majority of these patients should have the possibility of reconditioning through both central and peripheral adaptation towards an increased aerobic capacity. ${ }^{1,2}$ However, of obvious reasons such individually adapted exercise programmes are difficult to perform in these patients, since the balance towards disuse and overuse is very narrow. ${ }^{9,10}$ The present results indicate that advises of more rest and adaptations should be followed by parallel nutritional advises to avoid overweight.

New impairment of breathing is a matter of great concern to many polio survivors. In our study the number of patients who were in need of respiratory assistive devices increased from three to four (Figure 4), and the number of persons with FEV1 below 1,5 1 rise from six to ten (Table 2). However, as many as $33 \%$ (22 patients) reported increased breathing problems during the follow-up period (Figure 2). The latter symptom might be due to reduced cardiopulmonary fitness rather than to respiratory problems. Patients with major respiratory problems are usually not admitted to our rehabilitation hospital, but rather to a department of pulmonary diseases. Most of our patients had thus reasonably good lung function at both examinations (Table 2).

In the present study men had a more pronounced fall in max $\dot{V O} 2$ than women (Table 3), probably because they had had a relatively more reduced level of physical activity. Thus, men and women were at the similar low level of $\max \dot{V O} 2$ at the second examination. It is debatable if this activity level, and aerobic fitness level, are optimal.

The women reported more improvement in mental health than men during the period following the intervention program (Figure 1). Our explanation on this finding is that women experienced less emotional distress after having undergone a comprehensive evaluation and education programme. Even if their physical condition still worsened, they were relieved by knowing why they had new problems and that their new problems were possible to handle, and thus had less coping problems. The men in this study seemed to be more emotionally stable, and had thus less to gain mentally on the same intervention (Figure 1).

The majority of our patients reported increased muscle weakness, both in previously polio affected and not affected muscles (Figure 2). Objective strength measuring were only performed at the last examination in this study. However, other long-term follow-up studies of muscle strength have shown objective deterioration in post-polio cases, ${ }^{11-14}$ but even if the reduction in muscle strength was relatively small and comparable with normal reduction with increasing age, the reduction might have a substantial effect on polio patients with limited muscle reserve. Most polio patients can increase their muscle strength by training. ${ }^{15,16}$ However, it is a question whether intensive strength exercises might be dangerous on longer term, especially in patients with so-called unstable polio, ie with very large motor units. ${ }^{11,14,17,18}$

General fatigue was an increasing problem in $77 \%$ of the polio patients in this study (Figure 2). This symptom might partly be due to the low cardiopulmonary fitness and reduced neuromuscular function, but there is also theories on central polio virus affection. ${ }^{19-20}$ Our study cannot distinguish between central and peripheral causes for fatigue. However, according to depression scores performed in the patients at examination 2 they were not above average, indicating that depression is probably no significant reason for the fatigue.

Swallowing disorders as an increasing complication to longstanding polio is possibly a problem area that we should have attention towards, as $11 \%$ reported increased problems with swallowing in this group (Figure 2). Examination with videofluoroscopy could be chosen for selected cases.

During the 3-5 year follow-up a substantial number of patients with the postpolio syndrome were leaving their jobs due to their postpolio problems, especially among women (Figure 6), partly due to the increasing age, of course. In a Danish nation-wide 
survey from 1990 among 3607 polio subjects, the personal experience of a good health state was closely related to their capacity of work. ${ }^{21}$ Based upon that finding, we were aware of the importance in arranging flexible solutions related to the employment situation, especially combined with more help at home. However, our experience was also that some patients were lucky to have our support on leaving their job due to their health problems.

Dependence in personal activities of daily living is reported to be fairly rare in the polio population, ${ }^{22}$ whereas dependence and difficulties in instrumental activities of daily living (eg transportation, cleaning, shopping) are more common. ${ }^{22}$ At examination 2 in our study totally $10 \%$ of the patients were dependent in personal activities of daily living, and the majority were dependent in instrumental activities of daily living, such as housework and carrying (Figure 5). These figures are probably higher than in the general polio population, but indicate an increasing need of personal assistance for patients with postpolio syndrome.

In conclusion, the present study shows that patients with the postpolio syndrome experience further deterioration of subjective symptoms, physical disability and cardiorespiratory deconditioning over a period of 3-5 years, in spite of comprehensive, interdisciplinary intervention program. The deterioration of cardiorespiratory fitness was also found to be more pronounced than expected from normal ageing. Thus, based upon these results, the only measurable benefits of our rehabilitation programme were in moral and support.

\section{References}

1 Stanghelle JK, Festvåg L, Aksnes AK. Pulmonary function and symptom-limited exercise testing in subjects with late sequelae of poliomyelitis. Scand J Rehab Med 1993; 25: 125-129.

2 Astrand PO, Rodahl K. Textbook of work physiology. London: McGraw Hill, 1986.
3 Stanghelle JK, Rand-Hendriksen S. Treatment of patients with post-polio syndrome in a rehabilitation hospital. Tidsskr Nor Laegeforen 1991; 111: 1356 - 1357.

4 Stanghelle JK, Helseth R, Roaldsen KS, Rand-Hendriksen S. 42 patients with postpolio syndrome. Tidsskr Nor Laegeforen 1991; 111: $3159-3162$.

5 Halstead LS, Rossi CD. New problems in old polio patients. Results from a survey of 539 polio survivors. Orthopedics 1985; 8: $845-850$.

6 Halstead LS, Wichers DO. Research and clinical aspects of the late effects of poliomyelitis. Birth Defects 1987; 23: $301-312$.

7 Gulsvik A. Obstructive lung disease in an urban population. Oslo: Department of Lung Diseases, Rikshospitalet, The National Hospital, University of Oslo, Norway, 1979.

8 Borg G. Perceived exertion as an indicator of somatic stress. Scand J Rehabil Med 1970; 2: 92 - 98.

9 Grimby G, Einarsson G. Post-polio management. Crit Rev Phys Rehabil Med 1991; 2: 189-200.

10 Halstead LS, Grimby G, eds. Post-polio syndrome. Philadelphia: Hanley \& Belfus, 1995.

11 Grimby G, Hedberg M, Henning GB. Changes in muscle morphology, strength and enzymes in a 4-5 year follow-up of subjects with poliomyelitis sequelae. Scand J Rehabil Med 1994; 26: $121-130$

12 Dalakas MC et al. A long-term follow-up study of patients with post-poliomyelitis neuromuscular symptoms. $N$ Engl $\mathrm{J} \mathrm{Med}$ 1986; 314: $959-963$.

13 Agre JC et al. A comparison of symptoms between Swedish and American post-polio individuals and assessment of lower limb strength - a four-year cohort study. Scand J Rehab Med 1995; 27: $183-192$.

14 Stålberg E, Grimby G. Dynamic electromyography and muscle biopsy changes in a 4-year follow-up study of patients with a history of polio. Muscle \& Nerve 1995; 18(7): 699-707.

15 Einarsson G. Muscle conditioning in late poliomyelitis. Arch Phys Med Rehabil 1991; 72: $11-14$.

16 Feldman RM. The use of strengthening exercises in post-polio sequelae. Orthopedics 1985; 8: $889-890$.

17 Cashman NR et al. Late denervation in patients with antecedent paralytic poliomyelitis. N Engl J Med 1987; 317: 7-12.

18 Perry J, Barnes G, Gronley JK. The postpolio syndrome. An overuse phenomenon. Clin Orthop Rel Res 1988; 233: 145-162.

19 Bodian D. Histopathologic basis of clinical findings in poliomyelitis patients. Am J Med 1949; 6: $563-578$.

20 Bruno RL, Cohan JM, Galaski T, Frick NM. The neuroanatomy of post-polio fatigue. Arch Phys Med Rehabil 1994; 75: 498 - 504.

21 Lønnberg F. Late onset polio sequelae in Denmark. Sociomedical adaptation of polio survivors. Scand J Rehab Med 1993; suppl 28: $24-31$.

22 Grimby G, Jonsson AL. Disability in poliomyelitis sequelae. Physical Therapy 1994; 74(5): $415-424$ 